\title{
Aortic aneurysm with a ruptured dissection in a 15-year-old boy with hypoplastic left heart syndrome
}

\author{
Moritz Stolla, MD, ${ }^{\mathrm{a}}$ Ann Sweeney, MD, ${ }^{\mathrm{b}}$ George M. Alfieris, MD, ${ }^{\mathrm{b}}$ and Leon A. Metlay, MD, ${ }^{\mathrm{a}}$ \\ Rochester, NY
}

Hypoplastic left heart syndrome (HLHS) is a severe and fatal congenital heart defect that affects approximately 1000 newborns annually in the United States and is palliated through 3 consecutive surgical procedures. ${ }^{1}$ Postoperative mortality after the staged surgical palliation for HLHS has decreased as the field of congenital heart surgery has advanced. ${ }^{2}$ The first survivors are now in their third decade of life, and as the population increases, late-onset complications have been observed.

We report the case of a 15-year-old boy who developed an 8 -cm aneurysm in the ascending aorta that dissected from an intimal tear proximal of the innominate artery. A rupture of the dissection in the descending thoracic aorta led to exsanguination with subsequent cardiac arrest and death. With more patients with HLHS palliated through surgical revision and surviving into adulthood, the incidence of this complication is likely to increase.

\section{CLINICAL SUMMARY}

A 15-year-old boy with a medical history of HLHS was transferred to our pediatric cardiac intensive care unit with abrupt onset of severe chest pain. He had undergone a Norwood procedure stage I at 6 days of life. During the stage I palliation, the undersurface of the proximal transverse arch was incised, and this incision was carried down both proximally and distally toward the left side of the hypoplastic ascending aorta $(5 \mathrm{~mm})$ to the level of the sinotubular junction. The adjoining portion of the main pulmonary artery was anastomosed side to side to the hypoplastic ascending aorta at the level of the sinotubular junction. The remainder of the ascending aorta and transverse arch was reconstructed with cryopreserved thin pulmonary artery patch using 7-0 Prolene. At 5 months of life, the patient underwent a bidirectional Glenn procedure with homograft augmentation for bilateral pulmonary artery stenosis. He

\footnotetext{
From the Department of Pathology and Laboratory Medicine, ${ }^{a}$ Department of Pediatric Cardiothoracic Surgery, ${ }^{\mathrm{b}}$ University of Rochester Medical Center, Strong Memorial Hospital and Golisano Children's Hospital, Rochester, NY.

Disclosures: Authors have nothing to disclose with regard to commercial support.

Received for publication Sept 19, 2013; revisions received Nov 3, 2013; accepted for publication Nov 14, 2013; available ahead of print Jan 10, 2014.

Address for reprints: Moritz Stolla, MD, Department of Pathology and Laboratory Medicine, University of Rochester Medical Center, Strong Memorial Hospital and Golisano Children's Hospital, 601 Elmwood Ave, Rochester, NY 14642 (E-mail: moritz_stolla@urmc.rochester.edu).

J Thorac Cardiovasc Surg 2014;147:e35-6

$0022-5223 / \$ 36.00$

Copyright (c) 2014 by The American Association for Thoracic Surgery http://dx.doi.org/10.1016/j.jtcvs.2013.11.034
}

then underwent a third surgery that included a total caval pulmonary connection with an extracardiac $20-\mathrm{mm}$ polytetrafluoroethylene (Gore-Tex; WL Gore \& Associates, Inc, Flagstaff, Ariz) tube graft from the inferior vena cava to the pulmonary artery with a 4-mm punch fenestration at the age of 5 years. On annual follow-up with his pediatric cardiologist, he was noted to have a dilated ascending aorta that was not progressing in diameter (Figure 1, A). Before admission, persistent, excruciating chest pain radiating to the back, hypotension, and diaphoresis developed in the patient. He was seen at an outlying hospital where computed tomography imaging of the chest and abdomen revealed an aortic root aneurysm with dissection extending to the level of the renal arteries. He was subsequently transferred to the University of Rochester Medical Center. Antihypertensive medications were administered to maintain his systolic arterial blood pressure at less than $110 \mathrm{~mm} \mathrm{Hg}$. The patient was sent for cardiac magnetic resonance imaging to further evaluate the extent of the dissection. While in the magnetic resonance imaging suite, the patient became unresponsive and underwent unsuccessful resuscitation.

Autopsy was performed and confirmed a large aneurysm of the ascending aorta (Figure 1,B). An intimal tear was found at the ascending aorta proximal and 180 degrees from the take-off of the innominate artery where the homograft would have been (Figure 1, $C$ and $E$ ). The resulting dissection propagated distally to the iliac artery bifurcation. It should be noted that the arch vessels had no evidence of dissection. The aortic rupture that ultimately led to death was located in the mid-thorax portion of the descending aorta (Figure 1,D). This caused a left hemothorax with $2.75 \mathrm{~L}$ of blood extracted at autopsy.

\section{CONCLUSIONS}

As surgical outcomes for palliation of HLHS are leading to better survival, late complications such as aortic dilation and aneurysm are likely to become more prevalent. ${ }^{3}$ Aortic aneurysms in surgically palliated patients with HLHS have been reported in prior case reports. ${ }^{4,5}$ To the best of our knowledge, this is the first report of dissection and subsequent rupture of the aorta in a child with HLHS. We hypothesize that the aorta is vulnerable to dissection when there is a rapid diameter change of the aortic lumen. In favor of this theory is also the development of substantial atherosclerotic plaque that was found in this patient at the level of the dissection, despite his young age (Figure 1, $B$ ), presumably due to persistent turbulent flow proximal 

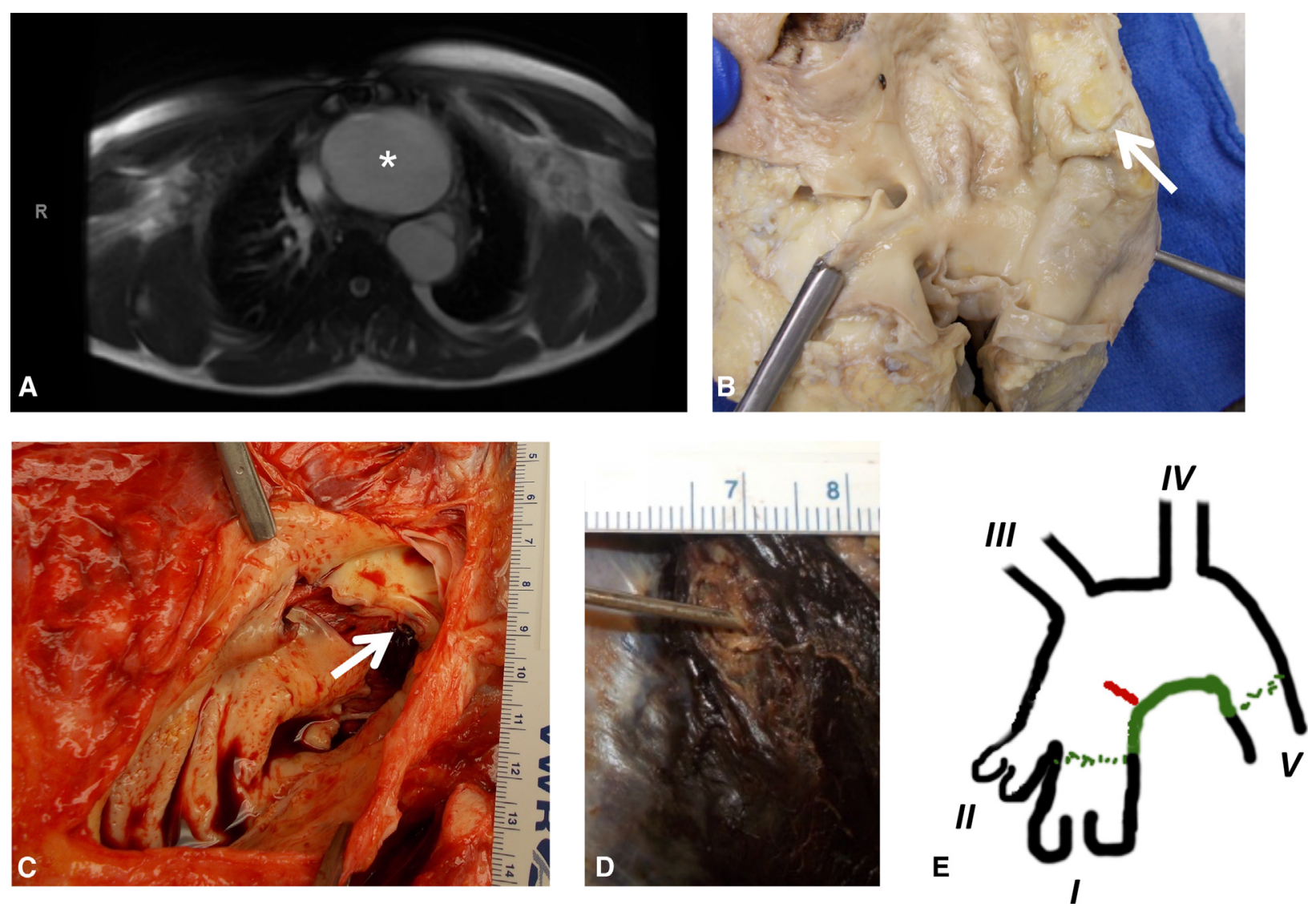

FIGURE 1. A, Computed tomography imaging of the thorax with contrast reagent showing a 7-cm aneurysm of the ascending neoaorta. Asterisk indicates vessel lumen. B, Post-formalin fixation image of the neoaortic aneurysm. White arrow indicates atherosclerotic plaques. C, Intra-postmortem image of the intimal tear in the area at the junction of the ascending neoaorta and the native aorta. White arrow indicates site of tear. D, Post-fixation image from outside of the new lumen aorta at the site of rupture (probe patent). E, Cartoon depicting the anatomy post-surgery and site of dissection. The green portion indicates the area of the patch, and the red line indicates the site of the dissection. Depicted in roman numerals are the following anatomic structures (clockwise): $\mathrm{I}=$ pulmonary valve, II = aortic valve, III = innominate artery, IV = left carotid artery, $\mathrm{V}=$ descending thoracic aorta.

to the tapering lumen. Because the intimal tear originated on the undersurface of the aortic arch where the homograft patch was placed, the injury could be related to a suture line or cannulation site.

\section{References}

1. Parker SE, Mai CT, Canfield MA, Rickard R, Wang Y, Meyer RE, et al. Updated National Birth Prevalence estimates for selected birth defects in the United States, 2004-2006. Birth Defects Res Part A Clin Mol Teratol. 2010;88:1008-16.

2. Czosek RJ, Anderson JB, Heaton PC, Cassedy A, Schnell B, Cnota JF. Staged palliation of hypoplastic left heart syndrome: trends in mortality, cost, and length of stay using a national database from 2000 through 2009. Am J Cardiol. 2013; 111:1792-9.

3. Cohen MS, Marino BS, McElhinney DB, Robbers-Visser D, van der Woerd W, Gaynor JW, et al. Neo-aortic root dilation and valve regurgitation up to 21 years after staged reconstruction for hypoplastic left heart syndrome. J Am Coll Cardiol. 2003;42:533-40.

4. Hebson CL, Kanter KR, Maher KO, Slesnick TC. Late development of a gigantic aneurysm of the neoaorta after Norwood palliation. Ann Thorac Surg. 2013;95: 1457.

5. Shuhaiber JH, Patel V, Husayni T, El-Zein C, Barth MJ, Ilbawi MN. Repair of symptomatic neoaortic aneurysm after third-stage palliation for hypoplastic left heart syndrome. J Thorac Cardiovasc Surg. 2006;131:478-9. 